Supplement of Biogeosciences, 18, 1823-1838, 2021 https://doi.org/10.5194/bg-18-1823-2021-supplement (c) Author(s) 2021. CC BY 4.0 License.

(c) (1)

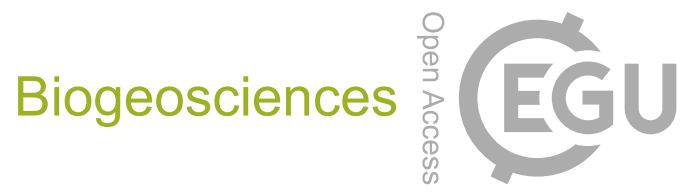

Supplement of

\title{
Warming and ocean acidification may decrease estuarine dissolved organic carbon export to the ocean
}

Michelle N. Simone et al.

Correspondence to: Michelle N. Simone (mnhsimone@gmail.com)

The copyright of individual parts of the supplement might differ from the article licence. 
Supplement:

Table S1. Measured total alkalinity (TA) and DIC used to calculate pH (Free scale) using $\mathrm{CO}_{2} \mathrm{SYS}$ directly compared to the measured $\mathrm{pH}$ from the cores using $\mathrm{HACH}$ multiprobe meter with $\mathrm{pH}$ probe. Mean absolute difference was used to estimate uncertainty in $p \mathrm{CO}_{2}$ calculations via $\mathrm{CO}_{2} \mathrm{SYS}$. Data used in a manuscript currently under review.

\begin{tabular}{|c|c|c|c|c|}
\hline $\mathrm{TA}(\mu \mathrm{M})$ & $\mathrm{DIC}(\mu \mathrm{M})$ & $\mathrm{pH}$ (calculated) & $\mathrm{pH}$ (measured) & absolute difference \\
\hline 2141.19 & 1932.80 & 8.06 & 8.09 & 0.03 \\
\hline 2197.35 & 1978.28 & 8.08 & 8.08 & 0.00 \\
\hline 2143.33 & 1933.06 & 8.07 & 8.08 & 0.01 \\
\hline 2240.94 & 2001.74 & 8.11 & 8.09 & 0.02 \\
\hline 2166.91 & 2006.67 & 7.94 & 7.92 & 0.02 \\
\hline 2209.33 & 2064.23 & 7.90 & 7.85 & 0.05 \\
\hline 2148.92 & 1967.39 & 7.99 & 7.94 & 0.05 \\
\hline 2257.76 & 2085.57 & 7.96 & 7.92 & 0.04 \\
\hline 2170.53 & 1982.56 & 8.00 & 7.98 & 0.02 \\
\hline 2198.29 & 2025.73 & 7.96 & 7.91 & 0.05 \\
\hline 2143.47 & 1952.13 & 8.01 & 7.98 & 0.03 \\
\hline 2237.67 & 2046.81 & 7.99 & 7.95 & 0.04 \\
\hline 2192.08 & 1967.57 & 8.05 & 8.10 & 0.05 \\
\hline 2183.91 & 1952.66 & 8.06 & 8.09 & 0.03 \\
\hline 2178.07 & 1954.05 & 8.05 & 8.09 & 0.04 \\
\hline 2219.11 & 1981.98 & 8.07 & 8.09 & 0.02 \\
\hline 2213.20 & 2043.19 & 7.92 & 7.89 & 0.03 \\
\hline 2194.63 & 2044.33 & 7.87 & 7.86 & 0.01 \\
\hline 2216.06 & 2028.52 & 7.96 & 7.89 & 0.07 \\
\hline 2232.28 & 2076.57 & 7.88 & 7.86 & 0.02 \\
\hline 2203.72 & 2006.91 & 7.98 & 7.97 & 0.01 \\
\hline 2185.34 & 2013.86 & 7.92 & 7.91 & 0.01 \\
\hline 2173.92 & 1964.42 & 8.01 & 8.00 & 0.01 \\
\hline 2225.09 & 2037.00 & 7.95 & 7.95 & 0.00 \\
\hline 2198.59 & 2096.54 & 7.79 & 7.82 & 0.03 \\
\hline 2258.32 & 2160.21 & 7.78 & 7.82 & 0.04 \\
\hline 2233.00 & 2141.65 & 7.76 & 7.80 & 0.04 \\
\hline 2200.45 & 2105.66 & 7.77 & 7.80 & 0.03 \\
\hline 2541.41 & 2435.35 & 7.77 & 7.60 & 0.17 \\
\hline 2290.22 & 2223.37 & 7.67 & 7.60 & 0.07 \\
\hline 2243.17 & 2180.95 & 7.66 & 7.60 & 0.06 \\
\hline 2220.31 & 2157.39 & 7.67 & 7.61 & 0.06 \\
\hline 2493.40 & 2356.90 & 7.84 & 7.68 & 0.16 \\
\hline 2261.24 & 2176.89 & 7.72 & 7.68 & 0.04 \\
\hline 2234.05 & 2138.49 & 7.76 & 7.71 & 0.05 \\
\hline 2204.96 & 2110.97 & 7.75 & 7.73 & 0.02 \\
\hline
\end{tabular}




\begin{tabular}{|c|c|c|c|c|}
\hline 2227.68 & 2113.68 & 7.77 & 7.81 & 0.04 \\
\hline 2234.09 & 2106.89 & 7.81 & 7.82 & 0.01 \\
\hline 2205.53 & 2085.13 & 7.79 & 7.83 & 0.04 \\
\hline 2250.33 & 2166.16 & 7.69 & 7.64 & 0.05 \\
\hline 2341.82 & 2215.36 & 7.80 & 7.63 & 0.17 \\
\hline 2256.07 & 2160.85 & 7.72 & 7.62 & 0.10 \\
\hline 2225.33 & 2141.49 & 7.70 & 7.62 & 0.08 \\
\hline 2242.14 & 2120.23 & 7.81 & 7.72 & 0.09 \\
\hline 2346.98 & 2172.34 & 7.92 & 7.71 & 0.21 \\
\hline 2255.08 & 2126.14 & 7.82 & 7.71 & 0.11 \\
\hline 2258.32 & 2091.91 & 7.91 & 7.77 & 0.14 \\
\hline 4477.45 & 4119.12 & 8.06 & 8.12 & 0.06 \\
\hline 4344.54 & 4009.16 & 8.04 & 8.10 & 0.06 \\
\hline 4465.28 & 4109.47 & 8.05 & 8.10 & 0.05 \\
\hline 4394.50 & 4048.37 & 8.05 & 8.11 & 0.06 \\
\hline 4339.08 & 4013.06 & 8.02 & 7.97 & 0.05 \\
\hline 4222.14 & 3934.02 & 7.98 & 7.96 & 0.02 \\
\hline 4359.09 & 4040.09 & 8.01 & 7.96 & 0.05 \\
\hline 4298.21 & 3979.27 & 8.01 & 7.97 & 0.04 \\
\hline 4318.94 & 3974.06 & 8.04 & 8.01 & 0.03 \\
\hline 4216.27 & 3898.43 & 8.01 & 7.97 & 0.04 \\
\hline 4339.12 & 4006.14 & 8.02 & 7.99 & 0.03 \\
\hline 4280.18 & 3943.36 & 8.03 & 8.01 & 0.02 \\
\hline 4291.14 & 3931.18 & 8.02 & 8.12 & 0.10 \\
\hline 4284.22 & 3928.93 & 8.02 & 8.11 & 0.09 \\
\hline 4266.35 & 3917.71 & 8.01 & 8.08 & 0.07 \\
\hline 4422.70 & 3958.88 & 8.13 & 8.11 & 0.02 \\
\hline 4218.32 & 3915.94 & 7.97 & 7.97 & 0.00 \\
\hline 4205.44 & 3913.13 & 7.96 & 7.95 & 0.01 \\
\hline 4187.63 & 3909.22 & 7.94 & 7.93 & 0.01 \\
\hline 4317.32 & 4000.77 & 7.98 & 7.96 & 0.02 \\
\hline 4187.10 & 3913.14 & 7.92 & 7.99 & 0.07 \\
\hline 4190.22 & 3876.99 & 7.97 & 7.99 & 0.02 \\
\hline 4422.61 & 3873.70 & 8.21 & 7.96 & 0.25 \\
\hline \multirow[t]{2}{*}{4310.09} & 3948.82 & 8.03 & 8.03 & 0.00 \\
\hline & & & Mean & 0.05 \\
\hline
\end{tabular}


Table S2. Overlapping mean control rates $( \pm \mathrm{SD})$ in current and high- $p \mathrm{CO}_{2}$ incubations for dark and light cycles. Units for dark and light rates $\left(\mu \mathrm{mol}-\mathrm{C}\right.$ or $\left.-\mathrm{O}_{2} \mathrm{~m}^{-2} \mathrm{~h}^{-1}\right)$ and net rates (mmol-C or $\left.-\mathrm{O}_{2} \mathrm{~m}^{-2} \mathrm{~d}^{-1}\right)$. Scaled means in Table S3 applied to significantly different means $(*)$ only.

\begin{tabular}{lllllll}
\hline & Dark & & Light & & Net \\
& Current & Future & Current & Future & Current & Future \\
\hline DIC & $\mathbf{1 1 9 9 . 8 9}$ & $\mathbf{1 0 7 7 . 5 3}$ & $\mathbf{- 8 6 0 . 7 9}$ & $\mathbf{- 6 1 2 . 9 4}$ & $\mathbf{4 . 0 7}$ & $\mathbf{5 . 5 7}$ \\
& $( \pm 149.43)$ & $( \pm 230.56)$ & $( \pm 218.92)$ & $( \pm 121.31)$ & $( \pm 2.18)$ & $( \pm 2.35)$ \\
DOC & $\mathbf{- 5 7 0 . 9 1}$ & $\mathbf{- 5 4 9 . 4 3}$ & $\mathbf{6 1 0 . 0 1}$ & $\mathbf{5 8 0 . 5 1}$ & $\mathbf{0 . 4 7}$ & $\mathbf{0 . 3 6}$ \\
& $( \pm 86.07)$ & $( \pm 7.76)$ & $( \pm 60.25)$ & $( \pm 116.67)$ & $( \pm 0.92)$ & $( \pm 1.47)$ \\
O2 & $\mathbf{- 1 0 1 8 . 0 2 *}$ & $\mathbf{- 7 8 7 . 0 7 *}$ & $\mathbf{7 0 0 . 2 0 *}$ & $\mathbf{5 2 5 . 4 7 *}$ & & \\
& $( \pm 38.20)$ & $( \pm 16.23)$ & $( \pm 11.66)$ & $( \pm 51.03)$ & & \\
P/R & & & & & $\mathbf{0 . 8 4}$ & $\mathbf{0 . 8 3}$ \\
& & & & $( \pm 0.01)$ & $( \pm 0.04)$ \\
\hline
\end{tabular}

Table S3. Scaled means $( \pm \mathrm{SD})$ for $\mathrm{R}$ and NPP rates $\left(\mu \mathrm{mol}-\mathrm{O}_{2} \mathrm{~m}^{-2} \mathrm{~h}^{-1}\right)$ under current and high- $p \mathrm{CO}_{2}$ incubations. CON* is the overlapping control present both weeks (note: current control and CON current are the same).

\begin{tabular}{|c|c|c|c|c|c|c|c|c|}
\hline \multirow[b]{2}{*}{ Offset } & \multicolumn{2}{|c|}{ Actual R } & \multicolumn{2}{|c|}{ Scaled R } & \multicolumn{2}{|c|}{ Actual NPP } & \multicolumn{2}{|c|}{ Scaled NPP } \\
\hline & & & rrent & 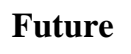 & & & & \\
\hline & $\begin{array}{l}-050.9 \\
( \pm 110.68)\end{array}$ & $( \pm 54.7)$ & $\pm 98.1)$ & $( \pm 62.8)$ & $( \pm 137.8)$ & $( \pm 138.9)$ & $( \pm 120.6)$ & $\begin{array}{l}\mathbf{6 6 . 0} \\
\pm 162.0)\end{array}$ \\
\hline & $\begin{array}{r}\mathbf{- 1 0 1 8 . 0} \\
( \pm 38.2)\end{array}$ & $\begin{array}{r}\mathbf{- 8 7 1 . 4} \\
( \pm 62.2)\end{array}$ & $( \pm 33.9)$ & $\begin{array}{l}\mathbf{- 9 9 9 . 2} \\
( \pm 71.3)\end{array}$ & $\begin{array}{l}700.2 \\
( \pm 11.7)\end{array}$ & $\begin{array}{l}902.9 \\
( \pm 41.2)\end{array}$ & $( \pm 10.2)$ & $\begin{array}{l}1053.0 \\
( \pm 48.0)\end{array}$ \\
\hline & $\begin{array}{r}-\mathbf{1 1 2 4 . 8} \\
( \pm 54.8)\end{array}$ & $\begin{array}{l}\mathbf{- 8 9 5 . 9} \\
( \pm 68.5)\end{array}$ & $\begin{array}{l}\mathbf{- 9 9 7 . 2} \\
( \pm 48.6)\end{array}$ & $\begin{array}{l}\mathbf{- 1 0 2 7 . 3} \\
( \pm 78.5)\end{array}$ & $\begin{array}{l}\mathbf{- 1 8 2 . 0} \\
( \pm 265.3)\end{array}$ & $\begin{array}{l}\mathbf{4 6 5 . 5} \\
( \pm 219.9)\end{array}$ & $\begin{array}{l}\mathbf{- 1 6 3 . 5} \\
( \pm 228.1)\end{array}$ & $\begin{array}{l}\mathbf{5 4 2 . 9} \\
( \pm 256.4)\end{array}$ \\
\hline$\Delta+5^{\circ} \mathrm{C}$ & $\begin{array}{l}\mathbf{- 1 3 1 5 . 8} \\
( \pm 85.1)\end{array}$ & $\begin{array}{l}\mathbf{- 1 0 2 4 . 4} \\
( \pm 62.7)\end{array}$ & $\begin{array}{l}\mathbf{- 1 1 6 6 . 4} \\
( \pm 75.5)\end{array}$ & $\begin{array}{l}\mathbf{- 1 1 7 4 . 7} \\
( \pm 71.9)\end{array}$ & $\begin{array}{l}-\mathbf{4 4 5 . 8} \\
( \pm 110.8)\end{array}$ & $\begin{array}{l}\mathbf{- 5 1 5 . 8} \\
( \pm 149.1)\end{array}$ & $\begin{array}{r}-390.1 \\
( \pm 96.9)\end{array}$ & $\begin{array}{l}\mathbf{- 6 0 1 . 6} \\
( \pm 173.8)\end{array}$ \\
\hline $\cos { }^{\circ}$ & $\begin{array}{l}\mathbf{- 1 0 1 8 . 0} \\
( \pm 38.2)\end{array}$ & $\begin{array}{r}-787.1 \\
( \pm 16.3)\end{array}$ & $\begin{array}{l}\mathbf{- 9 0 2 . 5} \\
( \pm 33.9)\end{array}$ & $\begin{array}{l}\mathbf{- 9 0 2 . 5} \\
( \pm 18.6)\end{array}$ & $\begin{array}{l}700.2 \\
( \pm 11.7)\end{array}$ & $\begin{array}{l}\mathbf{5 2 5 . 5} \\
( \pm 51.0)\end{array}$ & $\begin{array}{l}\mathbf{6 1 2 . 8} \\
( \pm 10.2)\end{array}$ & $\begin{array}{l}612.8 \\
( \pm 59.5)\end{array}$ \\
\hline
\end{tabular}

Table S4. Gross primary productivity (GPP) and productivity to respiration ratio (P/R) calculated for each temperature under both current and high- $p \mathrm{CO}_{2}$.

\begin{tabular}{lllll}
\hline & \multicolumn{2}{l}{ Current- $p$ CO } & \multicolumn{2}{l}{ High- $p$ CO } \\
& GPP & P/R & GPP & P/R \\
\hline$\Delta-3{ }^{\circ} \mathbf{C}$ & $\mathbf{1 3 5 1 . 9 5}$ & $\mathbf{0 . 8 9}$ & $\mathbf{1 6 0 5 . 8 2}$ & $\mathbf{1 . 0 9}$ \\
& $( \pm 185.26)$ & $( \pm 0.07)$ & $( \pm 111.28)$ & $( \pm 0.16)$ \\
Control & $\mathbf{1 5 1 5 . 3 8}$ & $\mathbf{0 . 8 4}$ & $\mathbf{2 0 5 2 . 2 3}$ & $\mathbf{1 . 0 3}$ \\
& $( \pm 37.02)$ & $( \pm 0.01)$ & $( \pm 108.95)$ & $( \pm 0.03)$ \\
$\Delta+\mathbf{3}^{\circ} \mathbf{C}$ & $\mathbf{8 3 3 . 6 9}$ & $\mathbf{0 . 4 2}$ & $\mathbf{1 5 7 0 . 1 4}$ & $\mathbf{0 . 7 7}$ \\
& $( \pm 182.21)$ & $( \pm 0.11)$ & $( \pm 267.41)$ & $( \pm 0.13)$ \\
$\Delta+\mathbf{5}^{\circ} \mathbf{C}$ & $\mathbf{7 7 6 . 2 9}$ & $\mathbf{0 . 3 3}$ & $\mathbf{5 7 3 . 0 8}$ & $\mathbf{0 . 2 5}$ \\
& $( \pm 172.36)$ & $( \pm 0.05)$ & $( \pm 133.81)$ & $( \pm 0.07)$ \\
\hline
\end{tabular}


Table S5. Dark and light fluxes of dissolved inorganic carbon (DIC) for each temperature under both current and highp $\mathrm{CO}_{2}$.

\begin{tabular}{lllll}
\hline & \multicolumn{2}{c}{ Current- $p \mathrm{CO}_{2}$} & \multicolumn{2}{c}{ High-pCO} \\
& \multicolumn{1}{c}{ Dark } & \multicolumn{1}{c}{ light } & \multicolumn{1}{c}{ Dark } & \multicolumn{1}{c}{ light } \\
\hline$\Delta-3^{\circ} \mathrm{C}$ & $\mathbf{6 7 6 . 5 4}$ & $\mathbf{- 5 5 5 . 0 1}$ & $\mathbf{8 3 6 . 9 7}$ & $\mathbf{- 6 3 5 . 2 9}$ \\
& $( \pm 224.38)$ & $( \pm 235.63)$ & $( \pm 117.45)$ & $( \pm 380.41)$ \\
$\mathbf{C O N}$ & $\mathbf{1 1 9 9 . 8 9}$ & $\mathbf{- 8 6 0 . 7 9}$ & $\mathbf{1 0 5 6 . 0 9}$ & $\mathbf{- 1 2 4 3 . 3 2}$ \\
& $( \pm 149.43)$ & $( \pm 218.92)$ & $( \pm 165.03)$ & $( \pm 162.03)$ \\
$\Delta+\mathbf{3}^{\circ} \mathbf{C}$ & $\mathbf{1 2 4 2 . 6 5}$ & $\mathbf{1 3 1 . 9 5}$ & $\mathbf{1 1 3 5 . 0 9}$ & $\mathbf{- 6 1 6 . 9 1}$ \\
& $( \pm 163.32)$ & $( \pm 73.50)$ & $( \pm 183.11)$ & $( \pm 87.93)$ \\
$\Delta+\mathbf{5}^{\circ} \mathbf{C}$ & $\mathbf{1 3 5 5 . 2 9}$ & $\mathbf{5 8 5 . 2 7}$ & $\mathbf{1 3 5 9 . 8 8}$ & $\mathbf{1 4 2 4 . 7 6}$ \\
& $( \pm 179.32)$ & $( \pm 363.97)$ & $( \pm 193.20)$ & $( \pm 202.41)$ \\
\hline
\end{tabular}

Table S6. Dark and light fluxes dissolved organic carbon (DOC) for each temperature under both current and highp $\mathrm{CO}_{2}$.

\begin{tabular}{lllll}
\hline & \multicolumn{2}{c}{ Current- $p \mathrm{CO}_{2}$} & \multicolumn{2}{c}{ High-pCO 2} \\
& \multicolumn{1}{c}{ Dark } & \multicolumn{1}{c}{ Light } & \multicolumn{1}{c}{ Dark } & \multicolumn{1}{c}{ Light } \\
\hline$\Delta-\mathbf{3}^{\circ} \mathbf{C}$ & $\mathbf{3 2 0 . 9 3}$ & $\mathbf{- 0 . 6 4}$ & $\mathbf{- 1 2 2 3 . 8 8}$ & $\mathbf{4 3 . 3 1}$ \\
& $( \pm 64.79)$ & $( \pm 3.98)$ & $( \pm 227.07)$ & $( \pm 299.53)$ \\
$\mathbf{C O N}$ & $\mathbf{- 5 7 0 . 9 1}$ & $\mathbf{6 1 0 . 0 1}$ & $\mathbf{- 7 6 0 . 1 4}$ & $\mathbf{- 1 4 8 . 2 4}$ \\
& $( \pm 86.07)$ & $( \pm 60.25)$ & $( \pm 237.39)$ & $( \pm 537.23)$ \\
$\Delta+\mathbf{3}^{\circ} \mathbf{C}$ & $-\mathbf{4 8 0 . 3 4}$ & $\mathbf{1 8 9 . 3 8}$ & $\mathbf{- 1 1 8 2 . 0 4}$ & $\mathbf{- 4 0 5 . 1 7}$ \\
& $( \pm 381.25)$ & $( \pm 238.78)$ & $( \pm 183.18)$ & $( \pm 820.27)$ \\
$\Delta+\mathbf{5}^{\circ} \mathbf{C}$ & $\mathbf{- 7 1 9 . 9 6}$ & $\mathbf{- 1 5 . 8 4}$ & $\mathbf{- 8 8 1 . 3 9}$ & $\mathbf{- 7 8 2 . 0 0}$ \\
& $( \pm 380.98)$ & $( \pm 14.23)$ & $( \pm 352.69)$ & $( \pm 340.26)$ \\
\hline
\end{tabular}




\section{Supplementary methods:}

Dissolved inorganic nitrogen (DIN) samples were collected at the start and end of the flux incubations and syringefiltered $(0.45 \mu \mathrm{m}$ cellulose acetate) into duplicate $10 \mathrm{~mL}$ polyethylene vials with a headspace, and stored frozen. Samples were analysed colorimetrically using a Lachat ${ }^{\mathrm{TM}}$ flow-injection system as described in Eyre and Pont (2003).

Table S7. DIN concentrations $(\mu \mathrm{M})($ mean \pm SD) at the start (minimum) and end of the full incubation cycle.

\begin{tabular}{lllll}
\hline Treatment & \multicolumn{2}{c}{ Current- $p \mathrm{CO}_{2}$} & \multicolumn{2}{c}{ High- $p \mathrm{CO}_{2}$} \\
& \multicolumn{1}{c}{ Start } & \multicolumn{1}{c}{ End } & \multicolumn{1}{c}{ Start } & \multicolumn{1}{c}{ End } \\
\hline$\Delta-3{ }^{\circ} \mathbf{C}$ & $\mathbf{1 . 1 9}$ & $\mathbf{2 . 0 2}$ & $\mathbf{1 . 8 5}$ & $\mathbf{6 . 6 6}$ \\
& $( \pm 0.01)$ & $( \pm 0.45)$ & $( \pm 0.27)$ & $( \pm 1.36)$ \\
\multirow{3}{*}{ Control } & $\mathbf{1 . 8 5}$ & $\mathbf{4 . 0 0}$ & $\mathbf{2 . 4 2}$ & $\mathbf{6 . 1 1}$ \\
& $( \pm 0.16)$ & $( \pm 0.27)$ & $( \pm 1.01)$ & $( \pm 1.39)$ \\
$\Delta+\mathbf{3}^{\circ} \mathbf{C}$ & $\mathbf{1 . 8 8}$ & $\mathbf{4 . 4 7}$ & $\mathbf{1 . 9 7}$ & $\mathbf{9 . 6 1}$ \\
& $( \pm 0.42)$ & $( \pm 2.10)$ & $( \pm 0.31)$ & $( \pm 1.36)$ \\
$\Delta+5^{\circ} \mathbf{C}$ & $\mathbf{2 . 3 7}$ & $\mathbf{1 5 . 5 2}$ & $\mathbf{2 . 4 0}$ & $\mathbf{1 4 . 6 8}$ \\
& $( \pm 0.18)$ & $( \pm 1.81)$ & $( \pm 0.58)$ & $( \pm 4.42)$ \\
\hline
\end{tabular}

